\title{
Positive moments for scattering amplitudes
}

\author{
Brando Bellazzini, ${ }^{1,2}$ Joan Elias Miró, ${ }^{3}$ Riccardo Rattazzi, ${ }^{4}$ Marc Riembau, ${ }^{4,5}$ and Francesco Riva ${ }^{5}$ \\ ${ }^{1}$ Institut de Physique Théorique, Université Paris Saclay, CEA, CNRS, F-91191 Gif-sur-Yvette, France \\ ${ }^{2}$ CERN, Theoretical Physics Department, Rte de Meyrin 385, CH-1211 Geneva, Switzerland \\ ${ }^{3}$ International Centre for Theoretical Physics (ICTP), Strada Costiera 11, 34135 Trieste, Italy \\ ${ }^{4}$ Theoretical Particle Physics Laboratory (LPTP), Institute of Physics, EPFL, 1015 Lausanne, Switzerland \\ ${ }^{5}$ Départment de Physique Théorique, Université de Genève, \\ 24 quai Ernest-Ansermet, 1211 Genève 4, Switzerland
}

(Received 1 February 2021; accepted 22 June 2021; published 16 August 2021)

\begin{abstract}
We find the complete set of conditions satisfied by the forward $2 \rightarrow 2$ scattering amplitude in unitary and causal theories. These are based on an infinite set of energy dependent quantities (the arcs) which are dispersively expressed as moments of a positive measure defined at (arbitrarily) higher energies. We identify optimal finite subsets of constraints, suitable to bound effective field theories (EFTs), at any finite order in the energy expansion. At tree level arcs are in a one to one correspondence with Wilson coefficients. We establish under which conditions this approximation applies, identifying seemingly viable EFTs where it never does. In all cases, we discuss the range of validity in both energy and couplings, where the latter has to satisfy two-sided bounds. We also extend our results to the case of small but finite $t$. A consequence of our study is that EFTs in which the scattering amplitude in some regime grows in energy faster than $E^{6}$ cannot be UV completed.
\end{abstract}

DOI: 10.1103/PhysRevD.104.036006

Effective field theory (EFT) is the universal framework used to describe particle physics on the basis of the principles of quantum mechanics and relativity. The EFT construction is nicely independent of the detailed features of the microphysics lying above reachable energies. Yet unitarity, causality, and crossing place robust constraints on the structure of its couplings. In particular, these constraints take the form of sharp positivity bounds from dispersion relations for the scattering amplitude both forward [1-5], and at finite angle [6-10], with a multitude of interesting recent applications e.g., [11-24], in addition to the original studies in the context of the chiral Lagrangian [1-3].

In this article we extend the positivity conditions on the forward amplitude to what appears to be a complete set. This is made possible by: i) writing the dispersion relations in terms of suitable energy-dependent quantities in the EFT, the arcs, which are directly related to the Wilson coefficients at tree level and capture features of the RG evolution at the quantum level, and ii) noticing that by unitarity the arcs correspond to the sequence of moments of a positive measure over a compact interval. The resulting setup precisely fulfils the hypothesis of Hausdorff's

Published by the American Physical Society under the terms of the Creative Commons Attribution 4.0 International license. Further distribution of this work must maintain attribution to the author(s) and the published article's title, journal citation, and DOI. Funded by SCOAP ${ }^{3}$. moment problem, whose known solution provides the set of necessary and sufficient positivity constraints on the forward amplitude. We also extend some of our results beyond the forward limit, using the classic result in Refs. $[25,26]$ on the positivity of the $t$ derivatives of the imaginary part of the amplitude.

Our results are related and partly overlap with those obtained by the geometric approach put forward in Ref. [10]. An important difference is that our method enables to identify optimal constraints that involve a finite number of arcs/Wilson coefficients only. This is the situation closer to questions of phenomenological interest.

The constraints we present are most effective in derivatively coupled theories, where the forward amplitude is finite in the massless limit. There, the arcs are single scale quantities that purely depend on the running couplings, and our constraints directly bound the RG flow (an aspect briefly touched in Refs. $[4,17,18,20,21])$. One well formulated hypothesis that can be tested in this context concerns the existence of symmetries-exact, accidental or weakly broken-which may appear in the low-energy EFT. In particular, the nonlinear transformations

$\phi(x) \rightarrow \phi(x)+b+b_{\mu_{1}} x^{\mu_{1}}+\cdots+b_{\mu_{1} \cdots \mu_{N}} x^{\mu_{1}} \cdots x^{\mu_{N}}$,

with $b^{\prime} s$ traceless, are symmetries in EFTs where interactions have many derivatives and, therefore, deliver soft amplitudes, i.e., amplitudes with a fast energy $E$ growth. 
The case $N=0$ is familiar; $U(1)$ Goldstone bosons with $2 \rightarrow 2$ amplitudes $\mathcal{M} \sim E^{4}$. The same behavior arises for the EFT of massless particles with spin, like for the EulerHeisenberg Lagrangian in QED, as well as the theories of Refs. [27-29]. The $N=1$ case corresponds to Galileons [30], with $\mathcal{M} \sim E^{6}$, and so on. We refer to theories with large exponents in $\mathcal{M} \sim E^{2 n}, n>2$, as super-soft [5,31]. Similarly, longitudinal polarizations in theories with massive spin- $J$ particles have super-soft amplitudes with $2 n \geq 3 J$ [20]. For example, approximate linear diffeomorphisms suppress the self-interactions from the Einstein-Hilbert term, relative to the super-soft linearized $(\text { Riemann })^{3}$ terms. Such a scenario for gravity is incompatible with tree-level UV completions [12]. In this work we will show that supersoftness in general cannot emerge, neither by structure nor by accident, from any reasonable, weakly or strongly-coupled UV completion.

This paper is organized as follows. In Sec. I, focusing on the forward limit, we construct the necessary general dispersion relations and derive optimal bounds on the arc variables. In Secs. II A and II B we apply our results, first assuming a weakly coupled UV completion and then lifting this assumption while focusing on the more specific case of an Abelian Goldstone boson. In Sec. III we consider a first foray of our methodology beyond the forward limit. Finally in Sec. IV we summarize our results and offer an outlook.

\section{ARCS AND THEIR CONSTRAINTS}

We study the $2 \rightarrow 2$ scattering amplitude $\mathcal{M}$ of a single particle of mass $m$ (including the limit $m^{2} \rightarrow 0$ if it exists). We discuss first the forward limit $t \rightarrow 0$ in theories where it is finite, and define $\mathcal{M}(s) \equiv \lim _{t \rightarrow 0} \mathcal{M}(s, t)$; for concreteness we focus on the spin- 0 case, but our results carry over to arbitrary spin and flavor structure [5]. Extensions to $t \neq 0$ are postponed to Sec. III. We will assume the following analytic properties of $\mathcal{M}$ :

(a) Crossing symmetry in $s \leftrightarrow u$ with real analyticity, namely $\mathcal{M}(s)=\mathcal{M}^{*}\left(4 m^{2}-s^{*}\right)$.

(b) The singularities of $\mathcal{M}(s)$ in the complex $s$-plane consist solely of a unitarity cut at physical energies $s>4 m^{2}$, plus the crossing symmetric one at $s<0$, see e.g., Ref. [32]. ${ }^{1}$

(c) Unitarity, via the optical theorem, implies $\operatorname{Im} \mathcal{M}(s)>0$.

(d) The amplitude $\mathcal{M}(s)$ is polynomially bounded as $|s| \rightarrow \infty$. In particular, we assume $\mathcal{M}(s) / s^{2} \rightarrow 0$ as $s \rightarrow \infty$. In the gapped case, validity of this condition is ensured by the Froissart bound $[33,34]$.

Exploiting the analyticity of the amplitude in the upper half-plane, we define the arc variables (or simply "arcs")

\footnotetext{
${ }^{1}$ Resonances with masses $M^{2}<4 m^{2}$ below threshold, imply additional poles at $s=M^{2}, 3 M^{2}$. We ignore these for simplicity; their inclusion is straightforward.
}

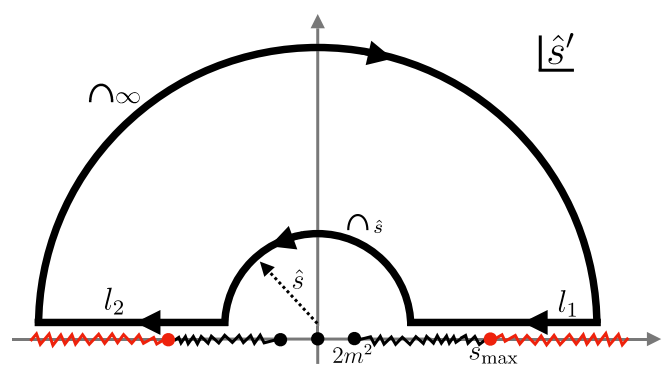

FIG. 1. The semicircle contour of Eq. (2) in the complex upper $\hat{s}^{\prime}$-plane. Wiggle lines denote the branch cuts on the real axis.

$$
a_{n}(\hat{s}) \equiv \int_{\bigcap_{\hat{s}}} \frac{d \hat{s}^{\prime}}{\pi i} \frac{\hat{\mathcal{M}}\left(\hat{s}^{\prime}\right)}{\hat{s}^{2 n+3}}
$$

where $\hat{s} \equiv s-2 m^{2}$ is the crossing-symmetric variable, $\hat{\mathcal{M}}(\hat{s}) \equiv \mathcal{M}(s)$, and $\cap_{\hat{s}}$ represents a counterclockwise semicircular path in the upper half-plane of radius $\hat{s}$, as shown in Fig. 1.

The Cauchy theorem implies that the integral over the closed contour $C=\cap_{\hat{s}}+\cap_{\infty}+l_{1}+l_{2}$ vanishes. Thus, we deform the integral in Eq. (2) along $\cap_{\hat{s}}$ into an integral along $\cap_{\infty}+l_{1}+l_{2}$. For integer $n$, we can use crossing symmetry and real analyticity to relate the amplitude above the left-hand cut (path $l_{2}$ ) to the one above the right-hand cut (path $l_{1}$ ),

$$
\hat{\mathcal{M}}(\hat{s}+i \epsilon)=\hat{\mathcal{M}}^{*}(\hat{s}-i \epsilon)=\hat{\mathcal{M}}^{*}(-\hat{s}+i \epsilon)
$$

Due to the Froissart bound, $\hat{\mathcal{M}} / \hat{s}^{2} \rightarrow 0$, the integral along the semicircle of infinite radius vanishes for $n \geq 0$. Thus, we can write Eq. (2) as

$$
a_{n}(\hat{s})=\frac{2}{\pi} \int_{\hat{s}}^{\infty} d \hat{s}^{\prime} \frac{\operatorname{Im} \hat{\mathcal{M}}\left(\hat{s}^{\prime}\right)}{\hat{s}^{\prime 2 n+3}}, \quad n \geq 0 .
$$

On the one hand, the arcs as defined via the IR representation [Eq. (2)] are IR quantities that can be systematically computed as an expansion in powers of $s$ in the domain of validity of the IR EFT $s \ll s_{\max }$, with $s_{\max }$ the cutoff. In the simple case of a tree-level amplitude in the forward limit, $\hat{\mathcal{M}}(\hat{s})=\sum_{n=0} c_{2 n} \hat{s}^{2 n}$ and the arcs match simply to Wilson coefficients, $a_{n}(\hat{s})=c_{2 n+2}$. Moreover, the scale dependence of arcs partly reflects the EFT RG flow, as we discuss in Sec. II.

On the other hand, according to the UV representation in Eq. (3), arcs receive contributions from all microphysics scales up to the far UV. So, Eq. (2) ideally represents something measurable in our low energy experiment, while the representation in Eq. (3) requires knowledge of the theory at all scales. Nevertheless, in this form, Eq. (3) has interesting properties that we now discuss. 


\section{A. All constraints}

From Eq. (3) it follows that the arcs are positive,

$$
a_{n}(\hat{s})>0,
$$

since the imaginary part of the forward amplitude is positive. In fact, convoluting in (3) a positive function $F$, we have

$$
\frac{2}{\pi} \int_{\hat{s}}^{\infty} d \hat{s}^{\prime} \frac{\operatorname{Im} \hat{\mathcal{M}}\left(\hat{s}^{\prime}\right)}{\hat{s}^{\prime 3}} F\left(\frac{\hat{s}}{\hat{s}^{\prime}}\right)>0 .
$$

For instance, $F\left(\hat{s} / \hat{s}^{\prime}\right)=\left[\hat{s} / \hat{s}^{\prime}\right]^{2 n}\left(1-\left[\hat{s} / \hat{s}^{\prime}\right]^{2}\right)$, with $\hat{s}<\hat{s}^{\prime}$, implies

$$
a_{n}-\hat{s}^{2} a_{n+1}>0 .
$$

Clearly, to every function $F$, positive in $\left(\hat{s} / \hat{s}^{\prime}\right)^{2} \in[0,1]$, there exist corresponding inequality constraints relating arcs of different orders. Characterizing the most general such function will allow us to address,

Question 1: What is the complete set of constraints the $\operatorname{arcs} a_{n}$ must satisfy?

To answer this question we will relate our problem to the theory of moments. The following change of variables

$$
x \equiv\left(\hat{s} / \hat{s}^{\prime}\right)^{2}, \quad d \mu(x)=\frac{d x}{\pi} \operatorname{Im} \hat{\mathcal{M}}(\hat{s} / \sqrt{x})
$$

simplifies the notation and defines a positive measure, so that we can write

$$
\hat{s}^{2 n+2} a_{n}=\int_{0}^{1} x^{n} d \mu(x) .
$$

A sequence of dimensionless numbers, defined as in Eq. (7) with $d \mu$ positive, is called a sequence of moments. In fact, we have a one-parameter family of sequences because each moment is a function of $\hat{s}$. We comment on the $\hat{s}$ dependence below.

We introduce the discrete derivatives

$$
(\Delta a)_{n}=\hat{s}^{2} a_{n+1}-a_{n},
$$

with higher order differences defined recursively, $\Delta^{k}=\Delta\left(\Delta^{k-1}\right)$ and $\Delta^{0} a_{n}=a_{n}$. For instance, $\left(\Delta^{2} a\right)_{n}=$ $a_{n}-2 \hat{s}^{2} a_{n+1}+\hat{s}^{4} a_{2+n}$, etc. A sequence of moments necessarily satisfies

$$
(-1)^{k}\left(\Delta^{k} a\right)_{n}=\frac{1}{\hat{s}^{2 n+2}} \int_{0}^{1} x^{n}(1-x)^{k} d \mu(x)>0
$$

since the functions

$$
F(x)=x^{n}(1-x)^{k}
$$

are positive in the whole integration domain. The case $k=1$ corresponds to Eq. (6).

The converse is also true, as implied by the Hausdorff moment theorem: if a sequence satisfies

$$
(-1)^{k}\left(\Delta^{k} a\right)_{n}>0 \quad \forall n, k \geq 0,
$$

then there exists a unique measure $d \mu$ such that Eq. (7) is satisfied. ${ }^{2}$ This theorem, following from the fact that the functions in Eq. (9)—called Bernstein polynomials—are a basis of all positive functions in $[0,1]$, provides an answer to our Question 1.

As mentioned above, both the arcs and their discrete derivatives depend explicitly on the scale $\hat{s}$, as captured by

$\frac{d}{d \hat{s}}\left[(-1)^{k} \Delta^{k} a_{n}\right]= \begin{cases}-\frac{2}{\pi} \frac{\operatorname{I} m \mathcal{M}\left(\hat{s}+2 m^{2}\right)}{\hat{s}^{3+2 n}} & (k=0) \\ 2 k \hat{s}\left[(-1)^{k} \Delta^{k-1} a_{n+1}\right] & (k \geq 1),\end{cases}$

which is negative for all $k$, because of Eq. (10). Therefore, as $\hat{s}$ is increased, the arcs decrease proportionally to $\operatorname{Im} \mathcal{M}$. This implies that, given an EFT, the constraints Eq. (10) for $k \geq 1$ become more stringent as $s$ increases. Conversely, if the conditions Eq. (10) are satisfied at one scale $\hat{s}$ they are automatically satisfied at smaller scales. This behavior will play an important role later on, when we discuss constraints on the arcs in specific EFTs.

\section{B. Optimal bounds for a finite set of arcs}

In practice we often focus on a finite number of arcs. For instance, in a typical EFT only the first few powers of $s$ are phenomenologically interesting — at tree-level this corresponds to the first few arcs. Thus it is natural to ask,

Question 2: Considering only a finite number $N$ of arcs, what are their optimal constraints?

Here optimal means the projection of all constraints on the finite set. In the language of the previous section, we ask: assuming the components of $\vec{a}=\left\{\hat{s}^{2} a_{0}, \ldots, \hat{s}^{2+2 N} a_{N}\right\}$ are moments, what is the subspace $A(N) \subset \mathbb{R}^{N}$ on which $\vec{a}$ takes values?

Of course Eq. (10) still holds and, for $k+n \leq N$; it involves only the first $N$ arcs. However, the constraints with $k+n \geq N$ imply additional conditions on the subset $n \leq N$. In what follows we will show a simple procedure to extract this information.

Similarly to Question 1-that implied finding the most general positive function in $[0,1]$-Question 2 requires

\footnotetext{
${ }^{2}$ Arcs probing the theory at finite $s$ are crucial for the mapping to moments on a compact interval (Hausdorff's problem). Instead, a sequence made of amplitude's residues at $s \rightarrow 0$ (equivalent to arcs with vanishing radius) see e.g., [35], maps to a noncompact domain (Stieltjes half-moment problem), and the solution is not unique.
} 
finding a parametrization for the most general polynomial $p(x)=\sum_{i=1}^{N} \alpha_{i} x^{i}$ of finite degree $\leq N$, positive in $x \in[0,1]$. Indeed, via Eq. (7), each such $p(x)$ leads to a condition on arcs,

$$
\int_{0}^{1} p(x) d \mu(x)>0 \Rightarrow \sum_{i=0}^{N} \alpha_{i} a_{i}>0 .
$$

One can prove that any such polynomial can be written as [36]

$$
p=\sum_{J} \underbrace{q_{J, 1}^{2}}_{\text {type-1 }}+\underbrace{x q_{J, 2}^{2}}_{\text {type-2 }}+\underbrace{(1-x) q_{J, 3}^{2}}_{\text {type-3 }}+\underbrace{x(1-x) q_{J, 4}^{2}}_{\text {type-4 }},
$$

where $q_{J, k}(x)$ 's are nonzero real polynomials (not necessarily positive) such that $p(x)$ is degree $N$, i.e., $q_{J, k}$ has at most degree $d_{k}, \quad$ with $d_{1}=\lfloor N / 2\rfloor, \quad d_{2}=d_{3}=$ $\lfloor(N-1) / 2\rfloor$, and $d_{4}=\lfloor(N-2) / 2\rfloor$, where $\lfloor k\rfloor$ is the integer part of $k \geq 0$. Since Eq. (13) is a sum over positive terms, it is sufficient to discuss them individually. Therefore we drop the index $J$ and consider one by one generic polynomials $q_{k}$ of each type- $k$ in Eq. (13),

$$
q_{k}(x)=\sum_{j=0}^{d_{k}} \alpha_{k j} x^{j}
$$

with arbitrary real coefficients $\alpha_{k j}$.

We define the Hankel matrix $\left(H_{N}^{\ell}\right)_{i j}=a_{i+j+\ell}$, for $i, j=0, \ldots,\lfloor(N-\ell) / 2\rfloor$, so that $H_{N}^{\ell}$ involves arcs up to $a_{N}$ for $N-\ell$ even, and $a_{N-1}$ for $N-\ell$ odd. For instance,

$$
H_{4}^{0}=H_{5}^{0} \equiv\left(\begin{array}{lll}
a_{0} & a_{1} & a_{2} \\
a_{1} & a_{2} & a_{3} \\
a_{2} & a_{3} & a_{4}
\end{array}\right)
$$

Now, polynomials of type-1 imply,

$$
\int_{0}^{1} q_{1}(x)^{2} d \mu(x)=\hat{s}^{2} \sum_{i, j=0}^{\lfloor N / 2\rfloor} \hat{s}^{2(i+j)} \alpha_{1 i} a_{i+j} \alpha_{1 j}>0 .
$$

Since the vector $\left\{\alpha_{10}, \alpha_{11} \hat{s}^{2}, \alpha_{12} \hat{s}^{4}, \ldots \alpha_{1, N / 2} \hat{s}^{N}\right\}$ is arbitrary, the following Hankel matrix must be positive definite,

$$
H_{N}^{0} \succ 0 \text {. }
$$

Following similar steps one finds that the positiveness of $\int_{0}^{1} x q_{2}^{2}(x) d \mu(x)>0, \quad \int_{0}^{1}(1-x) q_{3}^{2}(x) d \mu(x)>0$, and $\int_{0}^{1} x(1-x) q_{4}^{2}(x) d \mu(x)>0$ imply,

$$
\begin{gathered}
H_{N}^{1} \succ 0, \\
H_{N-1}^{0}-\hat{s}^{2} H_{N}^{1} \succ 0,
\end{gathered}
$$

$$
H_{N-1}^{1}-\hat{s}^{2} H_{N}^{2} \succ 0
$$

respectively. We refer to Eqs. (16)-(17) as homogeneous and Eqs. (18)-(19) as inhomogeneous constraints.

Since (13) is an arbitrary positive polynomial for $x \in[0,1]$, Eqs. (16)-(19) represent the optimal constraints, providing an answer to Question 2. For instance, Eqs. (16)-(19) with $N=2$ define the $A(2)$ region:

$$
\left(\begin{array}{ll}
a_{0} & a_{1} \\
a_{1} & a_{2}
\end{array}\right) \succ 0, \quad a_{1}>0, \quad a_{0}>\hat{s}^{2} a_{1}, \quad a_{1}>\hat{s}^{2} a_{2} .
$$

This is illustrated in the left panel of Fig. 2. The first constraint in (20) implies $a_{0} a_{2}>a_{1}^{2}$, and is saturated by the lowest parabola in Fig. 2; the fourth constraint in (20) is saturated by the upper line, the other constraints imply that the coordinates lie in the interval $[0,1]$. For comparison, the green lines show the constraints obtained using Eq. (10) and arcs up to $a_{2}$ (solid), $a_{3}$ (dashed), and $a_{4}$ (dotted), and then projected onto the $\left(s^{2} a_{1} / a_{0}, s^{4} a_{2} / a_{0}\right)$ plane: clearly these converge to the optimal result $\mathrm{A}(2)$.

Similarly, evaluating Eqs. (16)-(19) for $N=3$, we find $A(3)$ :

$$
\begin{array}{ll}
\left(\begin{array}{ll}
a_{0} & a_{1} \\
a_{1} & a_{2}
\end{array}\right) \succ 0, \quad\left(\begin{array}{ll}
a_{0}-a_{1} \hat{s}^{2} & a_{1}-a_{2} \hat{s}^{2} \\
a_{1}-a_{2} \hat{s}^{2} & a_{2}-a_{3} \hat{s}^{2}
\end{array}\right) \succ 0, \\
\left(\begin{array}{ll}
a_{1} & a_{2} \\
a_{2} & a_{3}
\end{array}\right) \succ 0, \quad a_{1}>\hat{s}^{2} a_{2},
\end{array}
$$

which we illustrate in the right panel of Fig. 2.

Equations (16)-(19) capture how the full constraint on the arc sequence is projected on the first $N$ arcs. Of course we can consider the projection on any subset. In particular for a sequence $a_{k}, \ldots, a_{N}$ that starts at $k \neq 0$, Eqs. (16)-(19) are generalized by the substitution $\ell \rightarrow \ell+k$, e.g., Eq. (16) becomes $H_{N}^{k} \succ 0$. Equation (6) belongs in this class.

To conclude this section we compare our results to those of Ref. [10], which-considering the forward amplitude-finds that consistent EFTs must satisfy the set of homogeneous Hankel matrix positivity constraints, Eqs. (16)-(17). Indeed, the whole set of homogeneous constraints (i.e., for arbitrarily large $N$ ) implies the ensemble of constraints in Eq. (10), ${ }^{3}$ and thus by the Hausdorff

\footnotetext{
${ }^{3}$ That is because, for $x \in[0,1]$, the Bernstein polynomials in Eq. (9) are arbitrarily well approximated by a combination of polynomials of type-1 and type-2 in Eq. (13), with arbitrarily large degree, which precisely correspond to the complete set of homogeneous constraints in Eqs. (16)-(17). For instance $F(x)=1-x$ is reproduced by $q_{1}(x)=\sqrt{1-x}=1-x / 2-$ $x^{2} / 8-x^{2} / 16-\cdots$ and $q_{2}=0$, and corresponds to an infinite Hankel matrix.
} 

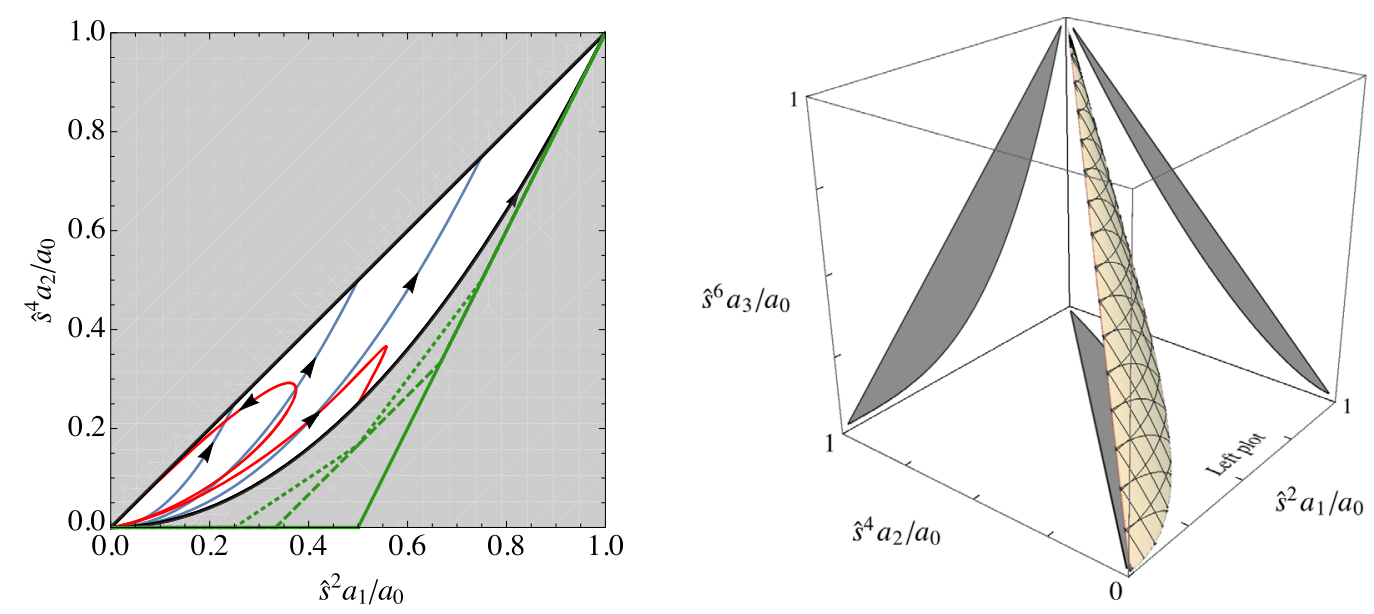

FIG. 2. Allowed regions for the arcs $a_{0}, a_{1}, a_{2}$ (Left) and including $a_{3}$ (Right), according to Eqs. (20), (21). Left: For fixed Wilson coefficients, as energy is increased, the theory spans a trajectory in the space of arcs: the blue trajectories (arrows in the direction of increasing $s$ ) correspond to examples in the weak coupling limit Eq. (23), the red trajectories are examples using Eq. (29) at strong coupling [with the explicit values Eq. (28) and large $g_{2}$ from Eq. (31)]. Values of $s^{4} a_{2} / a_{0}$ larger than the green solid/dashed/dotted lines are excluded by the conditions Eq. (10) from Bernstein polynomials, up to $k+n=2,3,4$ respectively. Right: The projections into twodimensional planes correspond to optimal bounds when only two coefficients are taken into account (the bottom projection corresponds to the left panel). The volume of the allowed region is $1 / 180$ with respect to the volume of the unit cube.

moment theorem is a necessary and sufficient set. However, when only a finite number $N$ of arcs/Wilson coefficients is considered, as it is often the case, our equations Eqs. (16)-(19) represent the optimal constraints. For instance, if we are interested in the allowed space for three arcs, as in Eq. (20), the first two homogenous conditions correspond to simple Hankel determinants and can be easily obtained with the methods of [10]. Instead, the latter two inhomogeneous conditions can only be obtained by considering infinite many homogeneous Hankel matrices.

\section{BOUNDS ON WILSON COEFFICIENTS}

Constraints on arcs translate, in principle, into constraints on the Lagrangian's Wilson coefficients. In practice, this translation is complicated by the fact that arcs might receive contributions from (infinitely) many Wilson coefficients. In what follows we discuss under which circumstances this translation is possible: Sec. II A discusses the tree-level approximation while Sec. II B discusses sizeable quantum effects.

\section{A. Tree level}

As a first application of our bounds, we focus on the forward limit in situations where we can consider just the IR tree level amplitude-we will discuss in the next section under which conditions this approximation holds. At low energy this takes a polynomial form ${ }^{4}$

\footnotetext{
${ }^{4}$ In the single flavor case the amplitude is a function of $s^{2}+t^{2}+u^{2}$ and $s t u$, and odd powers of $s$ vanish in the forward limit. See moreover footnote 1 .
}

$$
\hat{\mathcal{M}}(\hat{s})=\sum c_{d} \hat{s}^{d}=c_{0}+c_{2} \hat{s}^{2}+c_{4} \hat{s}^{4}+\cdots
$$

We compute the $a_{n}(\hat{s})$ using the definitions Eq. (2) and Eq. (22) and find,

$$
a_{n}=\left.\frac{1}{(2 n+2) !} \frac{\partial^{2 n+2}}{\partial \hat{s}^{2 n+2}} \hat{\mathcal{M}}(\hat{s})\right|_{\hat{s}=0}=c_{2 n+2}
$$

independently of $\hat{s}$. So the constraints of the previous sections can be read directly in terms of the coefficients appearing in the amplitude. From a practical point of view it is simpler to focus on a limited number of coefficients (rather than the complete series), so that Eqs. (16)-(19) represent the relevant constraints. Of these, the homogenous constraints of Eqs. (16)-(17) do not depend on $\hat{s}$ and thus represents properties of the UV theory that are intrinsic, i.e., independent of the overall scale of the dynamics. In particular they include Eq. (4), which implies that all coefficients $c_{n}$ be strictly positive [4].

On the other hand, the energy scale $\hat{s}$ appears explicitly in Eqs. (18)-(19) (this is translated in the normalization of Fig. 2 being $\hat{s}$-dependent). Given an EFT, in the form of a set of $c_{n}$ satisfying Eqs. (16)-(17), we can think of Eqs. (18)-(19) as defining the highest possible cutoff $\hat{s}_{\max }$ where new dynamics must modify our EFT amplitude.

For the simple case of the first three coefficients, in addition to positivity of each of them, Eq. (20) and Eq. (23) imply

$$
c_{2}-\hat{s}^{2} c_{4}>0, \quad c_{4}-\hat{s}^{2} c_{6}>0, \quad c_{2} c_{6}>c_{4}^{2} .
$$


For fixed values of the Wilson coefficients, as $\hat{s}$ increases, the arcs track trajectories in Fig. 2 (blue lines, for different values of $c_{n}$ ), that start for $\hat{s} \rightarrow 0$ at the origin and evolve along parabolas. In this case the cutoff must satisfy $\hat{s}_{\max }^{2}<c_{4} / c_{6}$.

The simplest inhomogeneous constraints $c_{2} \hat{s}^{2}-c_{n} \hat{s}^{n}>$ 0 , suffice to rule out any (even approximate) supersoft behavior, where the tree-level forward amplitude is dominated by the $O\left(s^{n}\right)$ growth, $n>2$. First of all, positivity already implies that supersoft symmetries Eq. (1) are never exact; they are always explicitly broken by a (possibly small) $c_{2}>0$. They could in principle have been appreciable in the regime of energy $\hat{s} \gtrsim\left(c_{2} / c_{n}\right)^{\frac{1}{n-2}}$. However, our bounds forbid that; super-soft theories cannot consistently be UV-completed at weak coupling, as the expansion in $s$ must be strictly decreasing $[19,20]$. We will see in the next section that the same conclusion holds also beyond the weak coupling approximation.

As a concrete example, consider for instance interactions of the form $(\partial \partial \phi)^{4}$, which is invariant under the Galilean symmetry $\phi \rightarrow \phi+b+b_{\mu} x^{\mu}$, and giving $\mathcal{M}(s) \sim s^{4}$. Purely on the basis of symmetries, these terms could have naturally dominated the more relevant $(\partial \phi)^{4}$ interactions, which break the Galilean symmetry. This is however inconsistent with the first inequality in (24) which forces $(\partial \partial \phi)^{4}$ to be subdominant.

\section{Faster $U V$ convergence}

So far the bounds in this section do not depend on $c_{0}$ in (22). This changes if we assume that $\lim _{s \rightarrow \infty} \hat{\mathcal{M}}(s) \equiv \mathcal{M}_{\infty}$ is finite. This is the case, for instance, if the theory in the $\mathrm{UV}$ is described by a finite number of resonances in the tree-level approximation.

For a finite $\mathcal{M}_{\infty}$ we can extend the definition of arcs in Eq. (2) to $n \geq-1$. Then we can repeat an analysis similar to the one that we did in the previous section for the subtracted amplitude $\hat{\mathcal{M}}-\mathcal{M}_{\infty}$, finding that the arcs define a sequence of positive moments $\left\{a_{-1}, a_{0}, a_{1}, \ldots\right\}$.

Note in particular that $a_{-1}=\hat{\mathcal{M}}(0)-\mathcal{M}_{\infty}$ can be regarded as the difference of effective couplings defined by the value of the forward amplitude in the IR and in the UV respectively, according to the weak coupling intuition. Since the first of the homogeneous conditions on the arcs is now $a_{-1}>0$, the IR forward amplitude is larger than the $\mathrm{UV}$ one. Moreover, $a_{-1} a_{1}-a_{0}^{2}>0$ must be satisfied and can be regarded as an upper bound on $c_{2}^{2} / c_{4}$ if the tree-level approximation of Eqs. (22)-(23) holds.

An interesting case that we consider in detail in the next section is the theory of a single Goldstone boson for which $c_{0}=0$. If the UV completion is perturbative $-\mathcal{M}_{\infty} \ll 16 \pi^{2}$, then we have

$$
0<\frac{c_{2}^{2}}{c_{4}} \leq-\mathcal{M}_{\infty} \ll 16 \pi^{2}
$$

on the Goldstone theory. ${ }^{5}$ The upper bound on $c_{2}^{2} / c_{4}$ is in agreement with the expectation that RG running effects on $c_{4}$ - that we will see Eq. (28) - are expected to be small in a weakly coupled theory.

\section{The boundary}

Which theories saturate Eqs. (16)-(19)? Hausdorff's theorem implies that the integration measure (the imaginary part of the UV forward amplitude) is uniquely determined if all arcs are known. If the measure has support on finitely many points, then a finite number of arcs suffice to determine the measure uniquely.

Physically, a measure that consists of $P$ distinct delta functions, $\operatorname{Im} \hat{\mathcal{M}}(\hat{s})=\pi / 2 \sum_{k=1}^{P} g_{k}^{2} M_{k}^{2} \delta\left(\hat{s}-M_{k}^{2}\right)$, is realized in the tree-level approximation when integrating out heavy particles with $P$ distinct masses $M_{1}<M_{2}<\ldots<M_{P}$ and effective squared couplings $g_{k}^{2}>0$. This situation corresponds to ${ }^{6}$

$$
a_{n}=\sum_{k=1}^{P} \frac{g_{k}^{2}}{M_{k}^{4 n+4}}
$$

These arcs lie at the boundary of the $A(2 P)$ region [defined by Eqs. (16)-(19) with $N=2 P$ ], as we now show.

In the variable $x \in[0,1]$ of Sec. IB, the measure $d \mu(x)$ consists of strictly positive delta functions located at $x_{k}=$ $\hat{s}^{2} / M_{k}^{4}$ for $k=1, \ldots, P$. In the EFT, i.e., $\hat{s}<M_{1}^{2}$, there are thus $P$ such deltas within $(0,1)$. Recalling Eq. (13), Hankel matrices of order $\bar{P}$ correspond to the quadratic forms generated by integrating $x^{a}(1-x)^{b} q_{\bar{P}-1}(x)^{2}$ in $d \mu(x)$, with $q_{\bar{P}-1}(x)$ a generic polynomial of order $\bar{P}-1$. This integral gives a vanishing result only if all the $\bar{P}-1$ zeroes of $q_{\bar{P}-1}(x)$ coincide with the $P$ deltas in the measure. This can only happen if $\bar{P}-1 \geq P$, in which case the corresponding Hankel matrix has order $>P$; Hankel matrices of order $\leq P$ are strictly positive definite, while they are only positive semidefinite if their order is $>P$. Considering then Eqs. (16)-(19), we conclude that, for $\hat{s}$ within the EFT, the arcs are in the interior of $A(N)$ for $N<2 P$ and at the boundary of $A(2 P)$.

This implies that-in weakly coupled theories-the measurements of the arcs in the IR allows to indirectly count the number $P$ of resonances in the UV; $P$ is just the smallest $\bar{P}$ for which $\operatorname{det} H_{2 \bar{P}}^{0}=0$.

\footnotetext{
${ }^{5}$ As an example, consider a potential $V=\lambda / 4\left(|\Phi|^{2}-v^{2}\right)^{2}$ for a canonically normalized complex scalar field $\Phi$. The tree-level forward scattering of the Goldstone bosons gives $\mathcal{M}(s)=$ $\left[s^{2} /\left(s+m_{h}^{2}\right)-s^{2} /\left(s-m_{h}^{2}\right)\right] /\left(2 v^{2}\right)$ where $m_{h}^{2}=\lambda v^{2}$. Therefore, $\mathcal{M}_{\infty}=-\lambda$ and $c_{n}=\lambda / m_{h}^{2 n}$, so that $c_{2}^{2} / c_{4}=\lambda$, which saturates the bound (25).

${ }^{6}$ For $P \rightarrow \infty$, the finiteness of $a_{0}$ requires that $g_{k}^{2} / M_{k}^{4 n+4}$ decays sufficiently fast as $k \rightarrow \infty$. Considering a large but finite number of particles is therefore a good approximation.
} 
The same reasoning as above also implies that for $\hat{s}=M_{1}^{2}$ (just at the edge of the EFT) the arcs are at the boundary of the $A(2 P-1)$ region. Indeed, when $\hat{s}$ approaches $M_{1}^{2}$ from below, $x_{1}=\hat{s}^{2} / M_{1}^{4}$ approaches the edge $x=1$ of the integration region, and the contribution of the lightest resonance drops out when integrated against the type-3 polynomial $(1-x) q_{\bar{P}-1}(x)^{2}$ of Eq. (13). Then, for $\bar{P}=P$ the zeros of $q_{\bar{P}-1}(x)$ can be chosen to coincide with the location of the remaining $P-1$ heavier resonances, hence $\operatorname{det}\left(H_{2 P-2}^{0}-M_{1}^{4} H_{2 P-1}^{1}\right)=0$. On the other hand, for $\bar{P}<P, q_{\bar{P}-1}(x)$ has fewer zeros than there are resonances. This implies that for $N<2 P-1$ Eq. (18) is still strictly satisfied for $\hat{s}=M_{1}^{2}$ and that $\hat{s}>M_{1}^{2}$ is needed in order to violate it; $N=2 P-1$ arcs allows an optimal estimate of the EFT cutoff $M_{1}$, while for $N<2 P-1$ the estimate is always suboptimal.

In fact, the whole UV spectrum and couplings can be extracted in the IR by determining the roots of the $P$ th order polynomial in $\hat{S}^{2}$ saturating Eq. (18), det $\left(H_{2 P-2}^{0}-\hat{s}^{2} H_{2 P-1}^{1}\right)=0 .^{7}$

As an example, consider two heavy particles $\varphi_{i=1,2}$ of masses $M_{i}$ and trilinear vertex $\frac{g_{i}}{\sqrt{2}}(\partial \pi)^{2} \varphi_{i} / M_{i}$, matching Eq. (26) - here $\pi$ is the massless state associated with the $2 \rightarrow 2$ amplitude. The first three $\operatorname{arcs} a_{0,1,2}$ populate the bulk of the allowed region $A(2)$ of Eq. (20) and Fig. 2, even for $\hat{s}=M_{1}$. An estimate of the cutoff using only these three arcs, $\hat{s}^{2}<a_{1} / a_{2}$, produces values that are always above the true cutoff $\hat{s}=M_{1}^{2}$. By instead considering four arcs $a_{0,1,2,3}$, the estimated cutoff becomes exact: Eq. (18) is satisfied for $\hat{s}=M_{1}^{2}$. Including five arcs, Eq. (16) is found to be marginally satisfied, i.e., $\operatorname{det} H_{4}^{0}=0$, thus determining the number of states.

\section{B. Beyond tree level}

Under which circumstances is the tree-level formula, $a_{n}=c_{2 n+2}$, a good approximation, and when do the conclusions of the previous section hold?

To answer this, we study an EFT including RG effects, restricting (for simplicity) to the massless case where $\hat{s}=s$. Moreover, in order for the forward limit to be well defined, we focus on the case of a derivatively coupled scalar, i.e., a Goldstone boson $\phi$ with symmetry $\phi \rightarrow \phi+b$. Indeed, the explicit computation that we present below-up to two loops and $O\left(s^{6}\right)$-does not exhibit any IR divergence.

\footnotetext{
${ }^{7}$ According to Eq. (26), the $k$ th resonance contributes to the $i j$ entry of any Hankel matrix a term proportional to $M_{k}^{-4(i+j)}$. The determinant associated to Eq. (18) is thus given by sum of terms $\epsilon_{i_{1} \ldots i_{P}} M_{k_{1}}^{-4 i_{1}} M_{k_{P}}^{-4 i_{P}}$ (hence fully antisymmetric in the $k_{i}$ ) weighed by the product of $g_{k_{i}}^{2}\left(1-\hat{s}^{2} / M_{k_{i}}^{4}\right)$. The sum over $i_{k}$ therefore vanishes for $s=M_{k^{2}}$ since only $P-1$ distinct $1 / M_{k_{i}}^{4}$ terms appear in the antisymmetric tensor. Then, the couplings $g_{k}^{2}$ can be extracted by solving Eq. (26) in terms of the arcs.
}

Moreover, for the Goldstone theory, the tree-level amplitude is finite at $t=0$, and divergences could originate only from collinear emissions (soft emission does not affect the total cross section and hence neither the imaginary part of the amplitude). Using collinear factorization (SCET, see e.g., [37]), it is easy to see that these are finite, as the positive powers of collinear momenta associated with the Goldstone derivative interactions always compensate putative divergences in collinear propagators. We therefore assume that the forward amplitude is well defined.

In the upper half-plane, up to $O\left(s^{6}\right)$, the most general Goldstone boson amplitude starts at order $O\left(s^{2}\right)$ and reads,

$$
\begin{aligned}
\mathcal{M}(s)= & c_{2} s^{2}+s^{4}\left[c_{4}+\beta_{4} \log (-i s)\right]-i \pi s^{5} \beta_{5} / 2 \\
& +s^{6}\left[c_{6}+\beta_{6} \log (-i s)+\beta_{6}^{\prime} \log ^{2}(-i s)\right]+O\left(s^{7}\right),
\end{aligned}
$$

where log is defined in the standard way, with the cut on the negative real semi-axis. Then we have $2 \log (-i s)=$ $\log (s)+\log (-s)$ for $\operatorname{Im} s \geq 0$ and $\log (s)-\log (-s)=i \pi$. For ease of notation we set the RG scale $\mu=1$, but the generic choice is reinstated through $\log (-i s) \rightarrow$ $\log \left(-i s / \mu^{2}\right), c_{n} \rightarrow c_{n}(\mu)$. An explicit calculation in the Goldstone case gives

$$
\begin{aligned}
& \beta_{4}=-\frac{7}{10} \frac{c_{2}^{2}}{16 \pi^{2}}, \quad \beta_{5}=+\frac{4}{15} \frac{c_{2} c_{2,1}}{16 \pi^{2}}, \\
& \beta_{6}=-\frac{83}{70} \frac{c_{4} c_{2}}{16 \pi^{2}}-\frac{1}{30} \frac{c_{2,1}^{2}}{16 \pi^{2}}-\frac{319}{175} \frac{c_{2}^{3}}{\left(16 \pi^{2}\right)^{2}}, \\
& \beta_{6}^{\prime}=\frac{83}{200} \frac{c_{2}^{3}}{\left(16 \pi^{2}\right)^{2}},
\end{aligned}
$$

where $c_{2,1}$ is the coefficient of $s^{2} t$ in the nonforward amplitude. At $O\left(s^{6}\right)$ in the energy expansion, Eq. (27) does not receive any further correction at any number of loops. From (27), the first three arcs read,

$$
\begin{aligned}
& a_{0}=c_{2}+\frac{s^{2}}{2} \beta_{4}+\frac{s^{3}}{3} \beta_{5}+\frac{s^{4}}{4}\left(\beta_{6}+\frac{\beta_{6}^{\prime}}{2}(4 \log s-1)\right)+\cdots \\
& a_{1}=c_{4}(s)+s \beta_{5}+\frac{s^{2}}{2}\left(\beta_{6}+\beta_{6}^{\prime}(2 \log s-1)\right)+\cdots \\
& a_{2}=-\frac{\beta_{4}}{2 s^{2}}-\frac{\beta_{5}}{s}+c_{6}(s)+\cdots
\end{aligned}
$$

where

\footnotetext{
${ }^{8} \mathrm{By}$ a slight abuse of notation, $c_{4}$ in Eq. (27) differs from the tree-level amplitude in Eq. (22) by a finite one-loop piece: $c_{4}^{(27)}=c_{4}^{(22)}+449 c_{2}^{2} /\left(300\left(16 \pi^{2}\right)\right)$.
} 


$$
\begin{aligned}
& c_{4}(s) \equiv c_{4}+\beta_{4} \log s \\
& c_{6}(s) \equiv c_{6}+\beta_{6} \log s+\beta_{6}^{\prime}\left(\log ^{2} s-\frac{\pi^{2}}{12}\right) .
\end{aligned}
$$

These functions are RG invariant by construction, and are the natural extensions of $c_{4,6}$ in the interacting theory.

The dots in Eq. (29) denote higher powers of $s$, of which there are a priori infinitely many. If all such contributions were important, the EFT would have no predictive power. A meaningful EFT exists only under certain assumptions on the convergence of the series. To this aim, a first unavoidable assumption, is the perturbativity of the dimensionless couplings $g_{n}^{2} \equiv c_{n}(s) s^{n}$ that control the IR loop expansion,

$$
g_{n}^{2} \equiv c_{n}(s) s^{n} \ll(4 \pi)^{2}
$$

This condition, which we assume throughout this work, implies that many of the higher order terms that (could) enter Eq. (29) must be small. For instance it implies that $\beta_{4} s^{2}$ and the contribution $\propto s^{4} c_{4} c_{2} / 16 \pi^{2}$ in $\beta_{6} s^{4}$ [see (28)] are subleading in $a_{0}$.

Nevertheless, Eq. (31) does not yet imply the validity of the tree-level approximation for the arcs. Indeed, the corrections to the tree-level result $a_{n}=c_{2 n+2}$, are controlled by a broader set of parameters given by the ratios

$$
\frac{\beta_{n} s^{n}}{c_{m}(s) s^{m}}
$$

We will refer to the situations where these parameters are small as strong perturbativity. For $n<m$ these parameters grow in the IR and become smaller in the UV, and vice versa for $n>m$; in the standard $R G$ parlance they respectively correspond to relevant and irrelevant deformations away from tree-level. For $n=m$, they capture instead the logarithmic RG running of Wilson coefficients [they measure the interaction strengths at the cutoffsee (25) for the weakly coupled case]. For instance, in the sigma-model example of Footnote $5, c_{n}=\lambda / m_{h}^{2 n}$ and $\beta_{n} \sim$ $\left(\lambda^{2} / 16 \pi^{2}\right) / m_{h}^{2 n}$ are characterized by one scale and one coupling so that the ratios in Eq. (32) are of order $\left(\lambda / 16 \pi^{2}\right)\left(s / m_{h}^{2}\right)^{n-m}$. Then, given $\lambda / 16 \pi^{2} \ll 1$ and $s / m_{h}^{2}<1$, for $n \geq m$ the parameters of Eq. (32) are always small (strongly perturbative). However, for $n<m$, they can be larger than unity at small enough energies,

$$
s \lesssim m_{h}^{2}\left(\frac{\lambda}{16 \pi^{2}}\right)^{\frac{1}{m-n}}
$$

In fact, these quantum effects always dominate the far IR $s \rightarrow 0$, where the arcs asymptote to

$$
a_{0} \rightarrow c_{2}, \quad a_{1} \rightarrow \beta_{4} \log s, \quad a_{n \geq 2} \rightarrow-\frac{\beta_{4}}{(2 n-2) s^{2 n-2}}
$$

For $c_{2}>0$, and given $\beta_{4}<0$, as implied by unitarity within the EFT

$$
0>-s^{-4} \frac{2}{\pi} \operatorname{Im} \mathcal{M}(s)=\beta_{4}+O(s),
$$

these arcs fulfill all the constraints. What happens is that for $s \rightarrow 0$, the arcs are fully dominated by the IR tail of the spectral density, which is positive and fully determined by the leading term $\propto c_{2}^{2}$ in the $2 \rightarrow 2$ cross section. The Hausdorff condition is then trivially satisfied and, as graphically represented in Fig. 2, all red trajectories flow to a common attractor as $s \rightarrow 0$.

When higher energies are considered, predictivity is only retained if contributions above a certain finite positive power of $s$ remain negligible, in particular when considering the arcs (29). In view of that, we will now discuss two scenarios for omitting higher order terms. We dub them Simplest EFT and Next-to-Simplest EFT. In the first case we assume that all irrelevant contributions to the arcs are negligible, namely

$$
\beta_{n} s^{n} \ll c_{m}(s) s^{m} \text { for } n>m \text {. }
$$

This is the standard situation in the context of EFTs. Instead, more complex scenarios arise by allowing irrelevant parameters to sizeably contribute to the arcs. In the Next-to-Simplest EFTs, we will allow

$$
\beta_{n} s^{n} \sim c_{m}(s) s^{m} \quad \text { for some } n>m,
$$

while all the other $\beta$ coefficients still fulfill Eq. (35).

\section{Simplest EFT}

The IR relevant contribution to the arcs drastically modify the allowed region for the running Wilson coefficients $c_{n}(s)$. However, this is not apparent in the first two arcs $a_{0}$ and $a_{1}$. Indeed, for the first arc alone we have $a_{0} \simeq c_{2}$, since there are no possible relevant nor marginal perturbations that enter. Therefore the positivity constraint on $a_{0}$ gives $c_{2}>0$-equivalent to the tree-level case.

Consider now the first two arcs. The size of $\beta_{4} / c_{4}$ controls the logarithmic running in $a_{1} \approx c_{4}(s)$. The bounds of Sec. I A, in particular the optimal bounds in Eqs. (16)-(19), read

$$
\begin{gathered}
c_{4}(s)>0 \\
c_{2}-c_{4}(s) s^{2}>0,
\end{gathered}
$$

in addition to $c_{2}>0$. Written in term of the running coefficient $c_{4}(s)$, Eqs. (37)-(38) have the same form as at 

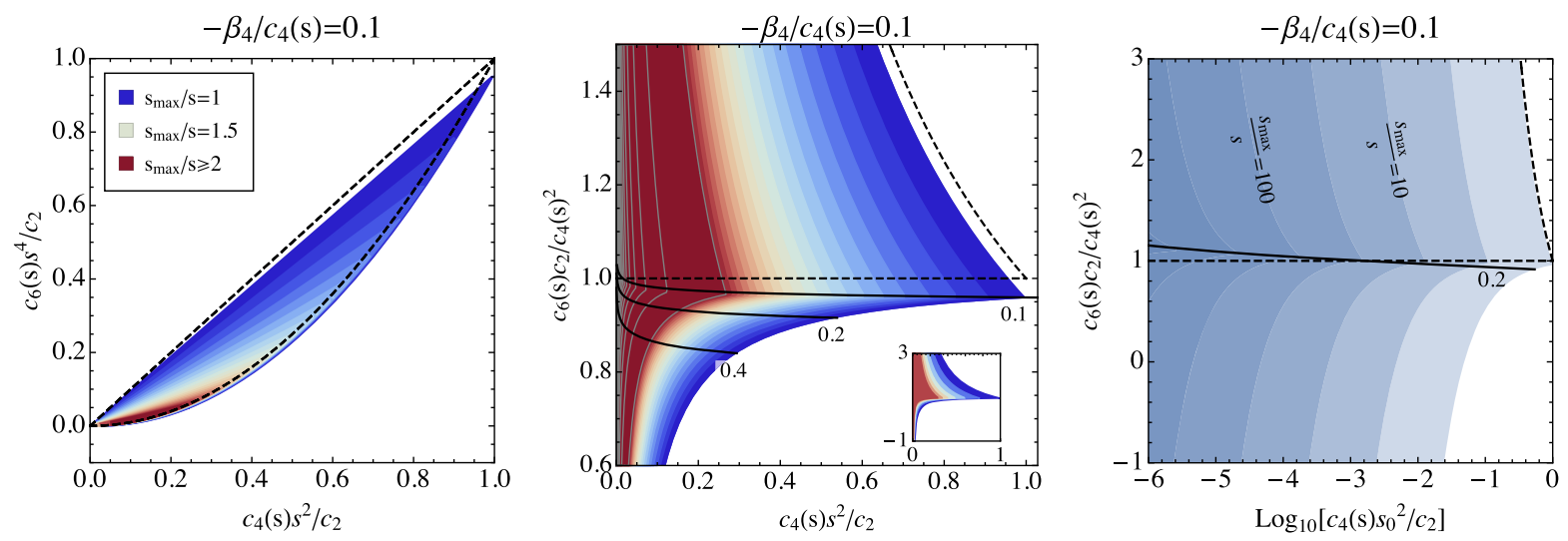

FIG. 3. In color the allowed area for (combinations of) Wilson coefficients $c_{2}, c_{4}(s)$ and $c_{6}(s)$, evaluated at a scale $s$. In all panels $\beta_{4} / c_{4}(s)=0.1$. Left and center panels: warmer colors denote points where the distance to the cutoff (i.e., the energy $s_{\max }$ where bounds are saturated) is larger; gray contour lines in the central plot have $s_{\max } / s=2,3,4, \ldots$ The black dashed curve denotes the tree-level expectation Eq. (24). The region above the black lines have $\beta_{4} / c_{6}\left(s_{\max }\right) s_{\max }^{2}<0.1,0.2,0.4$ respectively. The inset in the center panel shows a wider region of parameter space. Right panel: the same as in center panel but with logarithmic scale.

tree-level constraints of Eq. (24). Notice in particular that $c_{4}(s)$ (the lowest running Wilson coefficient) cannot be negative. Moreover, the second inequality, together with perturbativity defined in Eq. (31), justifies our neglect of the term $\propto s^{6} c_{4}^{2} / 16 \pi^{2} c_{2}$ in $\beta_{8} s^{8} / c_{2} s^{2}$. In other words, it implies that these terms automatically respect strong perturbativity (35).

In a causal and unitary theory, violation of any of Eqs. (37)-(38) must correspond to the failure of the hypothesis, Eq. (35). This implies that either the EFT is at its cutoff, or that it is nonstandard, in the sense that irrelevant higher derivative terms must be included, as we will discuss below in an example.

Starting with the third arc, the distinction between arcs and running Wilson coefficients becomes apparent. Indeed, the third arc $a_{2} \approx-\beta_{4} / 2 s^{2}+c_{6}(s),{ }^{9}$ includes a relevant deformation from its tree-level expectation $a_{2}=c_{6}$. The bounds read,

$$
\begin{gathered}
c_{4}(s)-c_{6}(s) s^{2}>-\frac{\beta_{4}}{2}, \\
c_{6}(s)-\frac{c_{4}(s)^{2}}{c_{2}}>\frac{\beta_{4}}{2 s^{2}} .
\end{gathered}
$$

Given $c_{4}(s)$ and $c_{6}(s)$ at an energy $s$, the first expression is stronger and the second is weaker than the tree-level conditions in (24). The weaker condition implies that the determinant $c_{2} c_{6}(s)-c_{4}(s)^{2}$, and indeed even $c_{6}(s)$, can be negative. In fact the RG effects in Eq. (30) alone, already violate the naive tree-level bounds; in the far IR $s \rightarrow 0, \mathrm{RG}$

\footnotetext{
${ }^{9}$ For simplicity we neglect $c_{2,1}$-this is consistent because $c_{2,1}$ is not renormalized by the other parameters. We include sizeable $c_{2,1}$ below, as a case study for the Next to Simplest EFT.
}

evolution leads to $c_{6}(s) \rightarrow \beta_{6}^{\prime} \log ^{2} s>0$, but makes the determinant negative,

$$
\operatorname{det}\left(\begin{array}{cc}
c_{2} & c_{4}(s) \\
c_{4}(s) & c_{6}(s)
\end{array}\right) \rightarrow\left(\beta_{4}^{2}-c_{2} \beta_{6}^{\prime}\right) \log ^{2} s<0,
$$

where we have used the explicit values for $\beta_{4}$ and $\beta_{6}^{\prime}$ from Eq. (28). Indeed it is now possible to have consistent EFTs where the determinant is so negative that, as energy increases, Eq. (40) is violated before Eq. (39); a radical difference with respect to the tree-level approximation, where only the inhomogeneous conditions depend on $s$. This is possible because quantum effects imply that arcs manifestly depend on the energy scale; Eq. (11) implies that all constraints become more stringent as $s$ increases, so that both homogeneous and inhomogeneous conditions play now a role in defining the theory's regime of validity.

This is illustrated in Fig. 3, where colored regions correspond to Eqs. (39), (40) and black dashed curves report the naive tree-level expectation.

We can therefore identify two interesting classes of theories. First, theories that possess a regime in which the parameters in Eq. (32) are small (in particular $\beta_{4} / c_{4} \ll 1$ and $\beta_{4} / c_{6} s^{2} \ll 1$ in the case of three arcs) and can be approximated by the tree-level expressions. Weakly coupled theories where the EFT is obtained by integrating out massive particles at tree level (like discussed on page 12), or at loop level (like for the Euler-Heisenberg Lagrangian), that belong in this class. ${ }^{10}$ In theories of this class, it is the inhomogeneous bounds [Eqs. (38)-(39) for three arcs] that are violated first, as $s$ increases.

\footnotetext{
${ }^{10}$ The discussion in Sec. II A corresponds to the idealized limit where the coupling goes to zero and the tree level regime for the arcs extends to arbitrarily small energies.
} 
This situation is illustrated in Fig. 3, where we have chosen $\beta_{4} / c_{4}(s)=0.1$, and where the region above the solid black lines correspond to varying sizes of $\beta_{4} / c_{6}\left(s_{\max }\right) s_{\max }^{2}$, as indicated in the figure.

On the other hand, in theories in which the relevant perturbation $\beta_{4} /\left(c_{6}(s) s^{2}\right)$ never becomes negligible, it is possible for $c_{2}, c_{4}(s), c_{6}(s)$ to lie at $O(1)$, outside of the tree-level naive boundary Eq. (24). In the central and right plots of Fig. 3, these theories feature (at all energy scales) a value of $c_{2} c_{6}(s) / c_{4}(s)^{2}$ significantly below its tree-level bound of 1 , and include the option of a negative value, implying $c_{6}(s)<0$. All in all, even in the Simplest EFT scenario, quantum effects open a qualitatively new region in parameter space. In these theories, as energy increases, it is the homogeneous conditions Eq. (40) that are saturated first. This is illustrated by the red trajectories in Fig. 2 exiting from the lower parabola.

\section{Next-to-simplest EFT}

We now study the simplest case in which there exists a sizeable effect associated with irrelevant parameters, as in Eq. (36) i.e., a sizeable $\beta_{n} s^{n} \sim c_{m} s^{m}$ for some $n>m$. (The Galileon limit, in which $c_{2,1} s \gg c_{2}$, is an example of this.) Since $c_{2,1}$ doesn't enter in the forward amplitude at tree level, it is not directly bounded by our discussion in Sec. II A (in Sec. III we discuss the amplitude away from the forward limit, but we anticipate that a positive $c_{2,1}$ is unbounded by those arguments). In this limit, the part of $\beta_{6}$ involving $c_{2,1}$, which we denote $\hat{\beta}_{6}=-c_{2,1}^{2} /\left(30\left(16 \pi^{2}\right)\right)$, can be potentially large and depart from strong perturbativity at sufficiently high energy within the EFT - though we still assume (strong) perturbativity for all higher coefficients.

Considering the first arc, keeping only the most important contributions in the Galileon limit $\left[\beta_{5}\right.$ in Eq. (II B) is suppressed with respect to $\hat{\beta}_{6}$ in this limit], positivity of $a_{0} \approx c_{2}+\hat{\beta}_{6} s^{4} / 4$ implies

$$
c_{2} \gtrsim s^{4} \frac{\left|\hat{\beta}_{6}\right|}{4},
$$

thus $c_{2,1}$ can be at most a loop factor larger than $c_{2}$, i.e., $c_{2,1} s^{2} \lesssim 8 \pi \sqrt{30 c_{2}}$, as already discussed in Refs. [5,7,15]. Equation (42) dictates that strong perturbativity between $\beta_{6}$ and $c_{2}$ can be violated only marginally.

Including also $a_{1} \approx c_{4}(s)+\hat{\beta}_{6} s^{2} / 2$, we find the further conditions

$$
-\frac{\hat{\beta}_{6}}{2} s^{4} \lesssim c_{4}(s) s^{2} \lesssim c_{2}-s^{4} \frac{\hat{\beta}_{6}}{4} .
$$

The first inequality implies that $c_{4}(s) s^{2}$ must still be positive. It also implies another bound of the form of Eq. (42), that can be written explicitly as

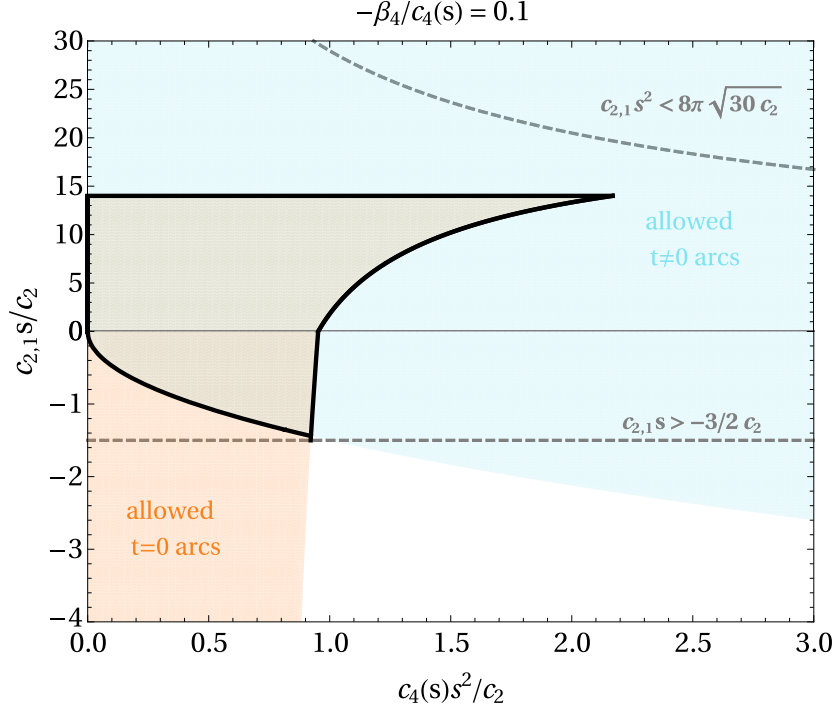

FIG. 4. The Galileon case $c_{2,1} \neq 0$, for fixed $-\beta_{4} / c_{4}(s)=0.1$. Orange Region: allowed from bounds on RGE of the forward $(t=0)$ amplitude (for comparison the upper dashed curve shows the upper bound from Ref. [15]). Blue region: allowed by the tree-level $t \neq 0$ bounds of Sec. III (for comparison the lower dashed curve reports the results of Ref. [8]). The solid black curve shows the intersection. Notice that upper and lower parts of the plot have different scales.

$$
c_{2,1} s \lesssim 8 \pi \sqrt{15 c_{4}(s)},
$$

and is stronger than the one implied by Eq. (42), for $c_{4}(s) s^{2}<2 c_{2}$.

The second inequality in Eq. (43) shows that $c_{4}(s) s^{2}$ can now be larger than $c_{2}$. However, compatibly with Eq. (42) means it cannot exceed $2 c_{2}$. Therefore the violation of the bound $c_{2}>c_{4}(s) s^{2}$ is only marginal, implying that supersoftness $c_{4}(s) s^{2} \gg c_{2}$ remains forbidden. These results are summarized in Fig. 4.

If we further include information for the third arc, $a_{2} \approx c_{6}(s)$ (where $c_{6}(s)$ is dominated by $\hat{\beta}_{6}$ and we neglect the term $\propto \beta_{4} / s^{2} \ll \hat{\beta}_{6}$ ), we have the inhomogeneous bound

$$
\frac{c_{6}(s) s^{4}}{c_{2}}\left(1+\frac{\hat{\beta}_{6} s^{4}}{2 c_{2}}\right) \gtrsim\left(\frac{c_{4}(s) s^{2}}{c_{2}}+\frac{\hat{\beta}_{6} s^{4}}{4 c_{2}}\right)^{2}
$$

So, a sizeable (negative) $\beta_{6}$ makes the lower bound on $c_{6}(s) s^{4} / c_{2}$ stronger, and shifts it towards larger values of $c_{4}(s) s^{2} / c_{2}$. These effects, however, appear in a relatively uninteresting regime of the theory. Indeed, the quantum effects $\propto \beta_{4} / s^{2}$ discussed above were sizeable in the IR and allowed for theories that depart from the tree-level approximation, while still being valid over a relatively large energy regime. Instead, the effects discussed here, are controlled 
by the $s^{2} \hat{\beta}_{6}$ term, that is sizeable only at high energy, when the theory is already close to the cutoff.

The couplings $c_{2,1}, c_{4}$ and $c_{6}$ are all compatible with Galilean symmetry; $c_{4}$ and $c_{6}$ are exactly invariant while $c_{2,1}$ is a sort of Wess-Zumino-Witten (WZW) term [30,38]. Instead, $c_{2}$ violates the symmetry. Our analysis shows that $c_{2,1}$ can be at most a loop factor larger than $c_{4}$, while the exactly invariant couplings $c_{4}$ and $c_{6}$ are directly limited by $c_{2}$. In some sense, our constraints privilege the WZW couplings over the exactly symmetric ones.

\section{BEYOND FORWARD}

In this section we extend our methodology to the class of amplitudes that are also analytic in $t$ in a finite region around $t=0$. This trivially includes the case of tree-level amplitudes generated by the exchange of massive states, but it also includes the general case of finite mass. Indeed, for fixed physical $s \geq 4 m^{2}$ the amplitude $\mathcal{M}(s, t)$ is analytic for complex $\cos \theta \equiv 1+\frac{2 t}{s-4 m^{2}}$ inside the so called Lehmann ellipse, namely an ellipse with foci at $\cos \theta= \pm 1$ [25] (see also e.g., [39]).

For $t$ finite and real, $s \leftrightarrow u$ crossing symmetry and real analyticity dictate $\mathcal{M}(s, t)=\mathcal{M}^{*}\left(4 m^{2}-s^{*}-t, t\right)$. Further constraints on the $t$ and $s$ dependence are given by the partial wave expansion, which diagonalizes the unitarity condition on the $S$-matrix. In the physical $s$-channel region the expansion reads

$$
\mathcal{M}(s, t)=\sum_{l=0}^{\infty} P_{\ell}(\cos \theta) f_{\ell}(s)
$$

while a similar relation also holds in the $u$-channel. Then, unitarity of the partial waves

$$
\operatorname{Im}\left(f_{\ell}(s)\right)>0 \quad \forall \ell \quad \text { for } s \geq 4 m^{2},
$$

together with positivity of the Legendre polynomials and their derivatives at $t=0$, Eq. (47) implies [26]

$\left.\partial_{t}^{k} \operatorname{Im} \mathcal{M}(s, t)\right|_{t=0}=\left.\sum_{\ell=0}^{\infty} \partial_{t}^{k} P_{\ell}(\cos \theta)\right|_{t=0} \operatorname{Im} f_{\ell}(s)>0$,

for all $k$ and for $s$ along the $s$-channel cut. The above additional positivity conditions can be exploited as we now discuss.

Considering the above properties we extend the definition of the arcs (2) to

$$
a_{n}(\hat{s}, t) \equiv \int_{\cap \hat{s}} \frac{d \hat{s}^{\prime}}{\pi i} \frac{\hat{\mathcal{M}}\left(\hat{s}^{\prime}, t\right)}{\left(\hat{s}^{\prime}+\frac{t}{2}\right)^{2 n+3}}
$$

where we recall $\hat{s} \equiv s-2 m^{2}, \hat{\mathcal{M}}(\hat{s}, t)=\mathcal{M}(s, t)$ and $\cap_{\hat{s}}$ is now a contour with radius $\hat{s}+t / 2$ centered at $-t / 2$.
The condition $\mathcal{M}(s, t)=\mathcal{M}^{*}\left(4 m^{2}-s^{*}-t, t\right)$ ensures the reality of the arcs. Furthermore using that $\hat{\mathcal{M}}(\hat{s}, t) / s^{2} \rightarrow 0$ for $s \rightarrow \infty$ (as dictated by the analog of the Froissart bound at finite $t$ [40]), the arcs can be expressed by a dispersive integral like in Eq. (3), ${ }^{11}$

$$
a_{n}(\hat{s}, t)=\frac{2}{\pi} \int_{\hat{s}}^{\infty} d \hat{s}^{\prime} \frac{\operatorname{Im} \hat{\mathcal{M}}\left(\hat{s}^{\prime}, t\right)}{\left(\hat{s}^{\prime}+\frac{t}{2}\right)^{2 n+3}} n \geq 0 .
$$

At this point we can take $t$-derivatives at $t=0$ and use Eq. (48) to obtain positivity conditions

$$
\begin{aligned}
\left.\partial_{t} a_{n}(\hat{s}, t)\right|_{t=0}= & a_{n}^{(1)}(\hat{s})-\frac{2 n+3}{2} a_{n+1 / 2}(\hat{s}), \\
\left.\partial_{t}^{2} a_{n}(\hat{s}, t)\right|_{t=0}= & a_{n}^{(2)}(\hat{s})-(2 n+3) a_{n+1 / 2}^{(1)}(\hat{s}) \\
& +\frac{(2 n+3)(2 n+4)}{4} a_{n+1}(\hat{s}),
\end{aligned}
$$

and so on; where we have defined

$$
a_{n}^{(k)}(\hat{s})=\frac{2}{\pi} \int_{\hat{s}}^{\infty} d \hat{s}^{\prime} \frac{\left.\partial_{t}^{k} \operatorname{Im} \hat{\mathcal{M}}(\hat{s}, t)\right|_{t=0}}{\hat{s}^{\prime 2 n+3}},
$$

and $a_{n}^{(0)} \equiv a_{n}$. Notice that while the arcs $a_{n}(\hat{s}, t)$ are defined for integer $n$ and can be written purely in terms of IR data according to Eq. (49), the $a_{n}^{(k)}(\hat{s})$ of (51), are defined for half-integer $n \geq 0$ and only through the UV representation in (52).

For every $k,\left\{\hat{s}^{2 n+2} a_{n}^{(k)}\right\}$, with half-integer $n \geq 0$, is a series of moments because the measure in (52) is positive according to (48). Therefore, they fulfill versions of the positivity constraints, analogous to Eqs. (16)-(19), including half-integer arcs. More precisely, half-integer arcs fulfill Eqs. (16)-(19), recast for new Hankel matrices $\left(h_{N}^{\ell}\right)_{2 i 2 j}=a_{i+j+\ell}$, where $2 i, 2 j=0,1, \ldots,\lfloor N-\ell\rfloor$ (here both $N$ and $\ell$ can be half-integers). The matrices $h_{N}^{\ell}$ contain both integer and half-integer arcs, e.g.,

$$
h_{1}^{0}=\left(\begin{array}{cc}
a_{0} & a_{1 / 2} \\
a_{1 / 2} & a_{1}
\end{array}\right) \text {. }
$$

Half-integer $\operatorname{arcs} a_{n+1 / 2}\left(n\right.$ integer) and all $a_{m}^{(k)}$ ( $m$ integer or half-integer for $k>0$ ) are not calculable in the EFT

\footnotetext{
${ }^{11}$ Since the amplitude is analytic also for $0 \leq t \leq 4 m^{2}$ [26], $\operatorname{Im} \hat{\mathcal{M}}(\hat{s}, t)=\left.\sum_{n} \partial_{t}^{n} \operatorname{Im} \mathcal{M}(s, t)\right|_{t=0} t^{n} / n !>0$ is positive there $[6,7]$, so that the constraints of Sec. I apply up to replacing $a_{n}(\hat{s}) \rightarrow a_{n}(\hat{s}, t)$ for $0 \leq t \leq 4 m^{2}$. In what follows we provide stronger constraints than these, by using that each derivative in Eq. (48) is separately positive. Notice also that it is in principle possible to build dispersion relations for other combinations that respect real analyticity, such as $\partial_{t} \hat{\mathcal{M}}-\partial_{\hat{s}} \hat{\mathcal{M}} / 2$. These also lead to Eq. (51).
} 
because they are defined in terms of UV integrals (52), and have no IR counterpart like (49). On the contrary, $a_{n}$ and $\partial_{t} a_{n}$, with integer $n$, can be computed directly within the IR. Our goal is therefore to understand the constraints on $a_{n}$ and $\partial_{t} a_{n}$ for arbitrary values of $a_{m}^{(k)}$ and $a_{n+1 / 2}$, compatible with their being moments. In Appendix, we outline an analytic procedure to do so, from which the bounds below are derived, but that applies in principle to all $N$. For more $t$ derivatives this procedure becomes cumbersome; in Ref. [41] we propose a numerical technique, based on semidefinite programming, to extract the bounds efficiently.

For instance, for $N=0$ we find the constraint on arcs' $t$ derivatives to be $\partial_{t} a_{0}>-\frac{3}{2} \frac{a_{0}}{\hat{s}}$, a condition that appears in a different form already in Ref. [8].

For $N=1$ our conditions constrain the space $\left\{\partial_{t} a_{0}, \partial_{t} a_{1}\right\}$ as

$$
\begin{aligned}
\partial_{t} a_{0} & >-\frac{3}{2} \sqrt{a_{0} a_{1}}, \quad \partial_{t} a_{1}>-\frac{5}{2} \frac{a_{1}}{\hat{s}}, \\
\partial_{t} a_{0}-s^{2} \partial_{t} a_{1} & >\frac{5}{2} \sqrt{\frac{a_{1}}{a_{0}}\left(\hat{s}^{2} a_{1}-\frac{3}{5} a_{0}\right),}
\end{aligned}
$$

which we illustrate in Fig. 5 for negative $\partial_{t} a_{0}$ (for $\partial_{t} a_{0}>0$, the allowed region is unbounded; the distance between the upper and lower boundaries of the projection on the $\left\{\hat{s}^{2} a_{1} / a_{0}, \hat{s}^{3} \partial_{t} a_{1} / a_{0}\right\}$ plane increases with $\left.\hat{s} \partial_{t} a_{0}\right)$. These conditions are more stringent than just $\partial_{t} a_{0}>-\frac{3}{2} \frac{a_{0}}{\hat{s}}$

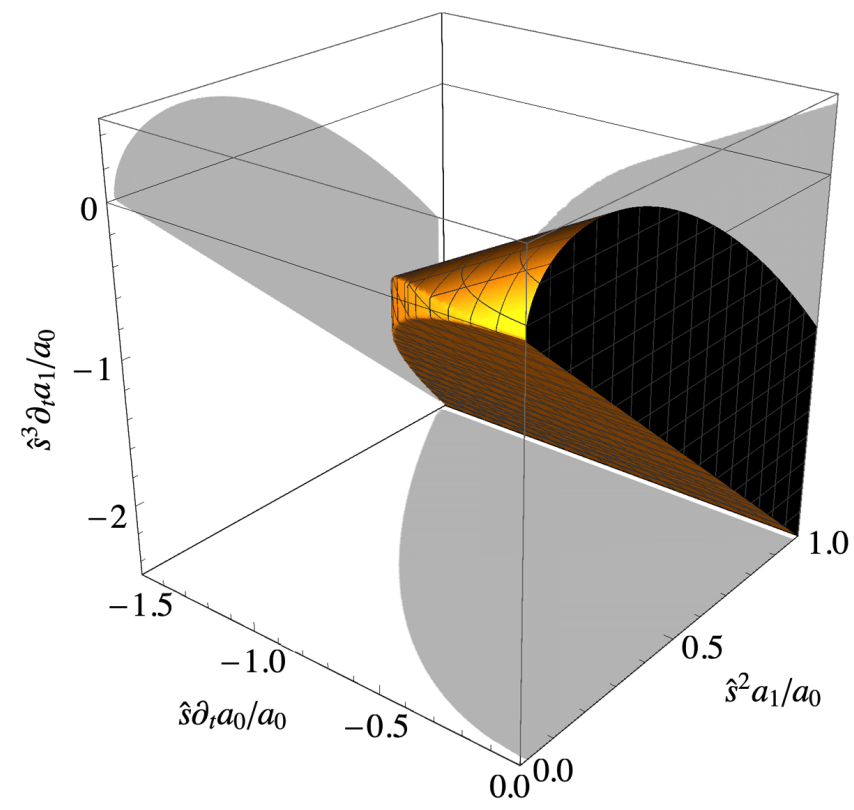

FIG. 5. Allowed region in the space of arcs and their first $t$-derivative for $\partial_{t} a_{0}<0$, according to Eq. (54); 2D projections in gray. At tree level we have $a_{0}=c_{2}, a_{1}=c_{4}, \partial_{t} a_{0}=c_{2,1}$ and $\partial_{t} a_{1}=c_{4,1}$. (which corresponds to the left boundary of the box in Fig. 5), as shown also in Fig. 4.

We illustrate an application of these bounds to the Wilson coefficients at tree level ${ }^{12}$

$$
\hat{\mathcal{M}}(\hat{s}, t)=\sum_{n+m>0} c_{n, m} \hat{s}^{n} t^{m} .
$$

Equation (54) reads at $t=0$,

$$
\begin{aligned}
c_{2,1} & >-\frac{3}{2} \sqrt{c_{4} c_{2}} \quad \hat{s} c_{4,1}>-\frac{5}{2} c_{4} \\
c_{2,1}-\hat{s}^{2} c_{4,1} & >\frac{5}{2} \sqrt{\frac{c_{4}}{c_{2}}}\left(\hat{s}^{2} c_{4}-\frac{3}{5} c_{2}\right)
\end{aligned}
$$

where we have identified $c_{n} \equiv c_{n, 0}$ to match the notation of the previous sections.

Equation (56) implies that $c_{2,1}$ can be negative but not arbitrarily so, as it is limited in magnitude by $\sqrt{c_{4} c_{2}}$.

To further illustrate the constraining power of Eq. (56), we can for instance use it to test the consistency of a theory where the amplitude is dominated by $\propto E^{10}$ terms for sufficiently large $E$ within the EFT domain of validity. By Lorentz invariance the only option is $\mathcal{M} \simeq \operatorname{stu}\left(s^{2}+t^{2}+u^{2}\right)$, corresponding to a $c_{4,1} s^{4} t$ term dominating $c_{2} s^{2}, c_{2,1} s^{2} t$ and $c_{4} s^{4}$. This hierarchy appears natural, since it is protected by one of the approximate symmetries in Eq. (1) (see [31]). Such an example is not constrained by our tree-level forward bounds in Sec. II A, simply because the largest contribution to the amplitude vanishes at $t=0$. However, Eq. (56) provides upper and lower bounds on $c_{4,1} \hat{s}^{5}$, controlled by more relevant Wilson coefficients (see Fig. 5 and its caption). In the physical region $|t|<s$, these bounds imply that $c_{4,1} s^{4} t$ can never dominate the terms with lower powers of $E$. In other words, supersoft amplitudes that vanish in the forward limit and have more powers of energy than $c_{2,1} s^{2} t$, are excluded by our bounds.

The same arguments lead to constraints on higher $t$ derivatives of arcs. The structure is always the same; $\partial_{t}^{k} a_{0}$ are bound from below, but can be arbitrarily large when positive. Instead $\partial_{t}^{k} a_{n}, n>0$ are bound from below and from above.

At tree level, this means that $c_{n, k}$, with $n>2$ for any $k$, fulfill two-sided bounds. Moreover, in the single flavor case

\footnotetext{
${ }^{12}$ At one loop, for a $U(1)$ Goldstone boson (i.e., with $c_{0}=0$ ), $\left.\partial_{t} \mathcal{M}\right|_{\substack{t \rightarrow 0 \\ m \rightarrow 0}}=c_{2,1} s^{2}-\frac{i 41 \pi c_{2}^{2}}{60\left(16 \pi^{2}\right)} s^{3}+\left(c_{4,1}-\frac{11 c_{2} c_{2,1}}{15\left(16 \pi^{2}\right)} \log (-i s)\right) s^{4}$
}

with $t \rightarrow 0$ before taking $m \rightarrow 0$, is finite. Therefore, for statements up to $O\left(s^{4} t\right)$, the use of the tree-level expressions is justified. We postpone a more refined discussion of these loop effects to Ref. [41]. 
TABLE I. Schematic summary of tree-level results. The gray area encompasses coefficients which are not independent because of crossing symmetry in the single-flavor case. $c_{0}$ is unconstrained, $c_{2}$ is constrained to be positive, $c_{2,1}$ is constrained to be larger than a combination of the other coefficients. All other coefficients are bounded below and above.

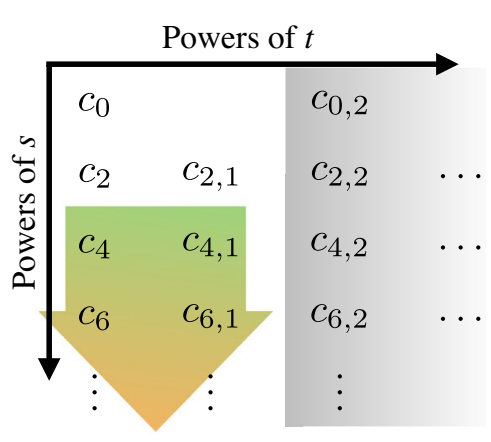

considered here, crossing symmetry implies that $c_{2, k}$ with $k \geq 2$ are related to $c_{2+k, 0}$ (for $k$ even) or $c_{1+k, 1}$ (for $k$ odd), which are already bounded from above from our constraints (gray area in Table I). For instance, the amplitude $\mathcal{M}(s, t) \propto\left(s^{2}+t^{2}+u^{2}\right)^{2}$ implies $c_{2,2}=3 c_{4}$, and $c_{4}$ is bounded from above. In other words, only $c_{2}$ and $c_{2,1}$ are unbounded from above by tree-level arguments; all other coefficients are instead bounded from below and above. We illustrate this in Table I.

As pointed out in Ref. [10], Eq. (48) contains more information than just positivity, which is what we have exploited so far. Indeed, the $\left.\partial_{t}^{k} \operatorname{Im} \mathcal{M}(s, t)\right|_{t=0}$ [and their integrals $\left.a_{n}^{(k)}(\hat{s})\right]$ are not merely positive, but are the sum of positive parameters with known coefficients; $\left.\left(s-4 m^{2}\right)^{k} \partial_{t}^{k} P_{\ell}(\cos \theta)\right|_{t=0}=(\ell+k) ! /(\ell-k) ! k !$. This implies more bounds involving at least three $a_{n}^{(k)}(\hat{s})$, with $k+n=$ constant [10]. Because they involve at least three different $t$ derivatives, and because they are saturated by the crossing symmetry condition in the simplest cases, these bounds didn't play a role in our discussion of supersoftness; for a relevant application see [42]. In Ref. [41] we will show how these bounds emerge in the language of moments and discuss quantitatively the impact of IR divergences.

\section{SUMMARY AND OUTLOOK}

In this paper we introduced a set of energy-dependent quantities, the arcs $a_{n}(s)$ of Eq. (2), that conveniently encode the constraints of causality, unitarity and crossing on the forward $2 \rightarrow 2$ scattering amplitude. A dispersion relation, Eq. (3), allows us to express the arcs as the moments of a positive measure in the range $[s, \infty)$. Hausdorff's moment theorem then establishes the set of necessary and sufficient conditions the arcs must satisfy, given the positivity of the measure. Both the arcs and the constraints are infinite sets. However we derive the projection of the full set of constraints on the subsets of the lowest arcs, $a_{0}, \ldots, a_{N}$ for any $N$. These are expressed by Eqs. (16)-(19) and fall into two classes, inhomogeneous and homogeneous, according to their explicit, or only implicit, dependence on $s$. These projections are interesting within EFTs, because the lowest $a_{n}$ 's encode the effects of the correspondingly lowest Wilson coefficients.

Our result is particularly relevant to determine the acceptable range of validity of EFTs in both couplings and energy. Concerning the latter and as implied by Eq. (11), the energy dependence of the arcs leads to stronger bounds on the EFT parameters as the energy is increased. Satisfaction of the positivity constraints at a certain $s$, automatically implies satisfaction at lower $s$ but not at higher $s$. When the parameters of a given EFT violate the constraints above a certain scale, the only option to respect positivity is the breakdown of the EFT description at or below that scale.

In the idealized limit where the tree level approximation holds exactly, the arc series is in one to one correspondence with the series of Wilson coefficients in the expansion of the forward amplitude. The energy dependence of the arcs arises at the quantum level from two sources: the RG evolution of the Wilson coefficients and collinear radiation from the initial state. The latter induces effects that typically go like powers of $\ln s / m^{2}$, and thus diverge in the massless limit, corresponding to the IR divergence of the total cross section. There are however situations, in particular when the interactions are purely derivative, where these IR divergences are absent and the arcs' energy dependence is purely controlled by $\mathrm{RG}$ evolution. It is this simpler situation that we have mostly considered for illustrative purposes, focusing on the theory of one Abelian Goldstone boson. We could have similarly considered the case of massless vectors or fermions, where gauge invariance or supersymmetry mandate derivative interactions.

The constraints are conveniently described by grouping EFTs into two broad classes. The first are EFTs that emerge from a weakly coupled UV completion, either at tree level or from loops. Here, for $s$ not too much below the physical EFT cutoff, the arcs are reliably approximated by the Wilson coefficients at tree level. Equations (16)-(19) then translate directly into sharp constraints on the Wilson coefficients. In particular, the energy dependent inhomogeneous constraints dictate the strict convergence of the $s$ expansion of the forward amplitude $\mathcal{M}(s)$ and rule out the possibility for supersoft EFTs, where $\mathcal{M}(s)$ grows faster than $s^{2}$. Indeed the energy-dependent constraints, through their violation, also allow us to infer the maximal cutoff of the EFT. As we discussed, even in these weakly coupled EFTs the tree level approximation for the arcs breaks down at sufficiently low $s$ because of mixing at the quantum level with more relevant Wilson coefficients. Consequently in the far IR the allowed region for the running Wilson coefficients differs significantly with respect to the near 
cutoff region. This fact is also directly related to the existence of the second class of EFTs, for which the tree level approximation for the arcs is never realized in their domain of validity. Positivity still implies strict constraints in coupling space and in particular rules out supersoft EFTs, at least under the simplest assumptions we could check. In this second class of theories the homogeneous constraints, now energy dependent because of quantum effects, can control the maximal allowed UV cutoff.

Our study mostly concerned the forward amplitude, but in Sec. III we extend it to $t \neq 0$. We studied the arc first $t$ derivative; in derivatively coupled theories, this too is free of IR divergences. The bounds we obtain complete our analysis into the realm of theories with suppressed forward amplitude. In particular they show that $\mathcal{M}(s)$ can be dominated over a limited range of energies by $\propto s^{2} t$ behavior, but that anything softer is forbidden. More bounds than those presented here can be derived using the explicit form of Legendre polynomials (in addition to positivity of their derivatives) [10], but always involve at least two $t$ derivatives. These involve quantities that are IR divergent in the $m \rightarrow 0$ limit, and go beyond the scope of the present study (see comments further down).

Our investigation could be furthered in a number of ways. One could be to try and connect to the $S$-matrix bootstrap [43-45]. This approach exploits the full $2 \rightarrow 2$ unitarity equation, schematically $2 \operatorname{Im} T>|T|^{2}$, while our analytical bounds purely exploit positivity $\operatorname{Im} T>0$. Besides trying to implement full unitarity one could perhaps use an ansatz for the $2 \rightarrow 2$ amplitude similar to the $S$-matrix bootstrap approach of [43].

An obvious way to extend our work would be to more systematically study other instances of derivatively coupled theories. In particular one could consider cases involving states of different helicity and with a flavor structure. Here it would be interesting to consider the forward amplitude for superpositions of helicity and flavor. A less obvious one would be to consider nonderivatively coupled EFTs where the cross section is affected by collinear divergences. In this case the bounds on the Wilson coefficients at some scale $s$ will seemingly have a dependence, to be determined, on the mass $m$ providing the IR regulation. Alternatively one could treat the mass $m$ itself as the RG scale and work with $s \sim m^{2}$.

\section{ACKNOWLEDGMENTS}

We thank Nima Arkani-Hamed, Thomas Becher, Gabriel Cuomo, Brian Henning, Yu-Tin Huang, Paolo Nason, and Andrea Wulzer for useful discussions, and Alberto Nicolis, Joao Penedones, and Francesco Sgarlata for comments on the manuscript. The work of M.R., F. R. and R. R. is supported by the Swiss National Science Foundation under Grants No. PP00P2-170578, No. 200021-178999, and No. 200020-169696 and through the National Center of Competence in Research SwissMAP. B. B. and F. R. were also supported by the Munich Institute for Astro- and Particle Physics (MIAPP) which is funded by the Deutsche Forschungsgemeinschaft (DFG, German Research Foundation) under Germany Excellence Strategy D EXC-2094 D 390783311. J.E. M., R. R., and F. R. thank the Simons Collaboration on the nonperturbative bootstrap for hospitality in ICTP-SAIFR.

\section{APPENDIX: ANALYTIC RESULTS AT FINITE $\boldsymbol{t}$}

It is convenient to rearrange the Hankel matrices $\left(h_{N}^{\ell}\right)$ defined above [Eq. (53)], in terms of blocks of either halfinteger or integer arcs, by extending the definition of Sec. I to $\left(H_{N}^{\ell}\right)_{i j}=a_{i+j+\ell}$ where now $\ell$ and $N$ can be half-integer or integer, and $i, j=0,1, \ldots,\lfloor(N-\ell) / 2\rfloor$, e.g.,

$$
H_{5 / 2}^{1 / 2}=\left(\begin{array}{ll}
a_{1 / 2} & a_{3 / 2} \\
a_{3 / 2} & a_{5 / 2}
\end{array}\right) .
$$

In this way, for $N$ integer, the constraints Eqs. (16)-(19) on $h_{N}^{\ell}$ (involving both integer and half-integer arcs) can be written in terms of constraints on $H_{N}^{\ell^{\prime}}$ (integer arcs only for $\ell^{\prime}$ integer) and $H_{N}^{\ell^{\prime}+1 / 2}$ (half-integer arcs only):

$$
\begin{aligned}
H^{1 / 2} & \succ 0, \quad H^{1} \succ H^{1 / 2}\left(H^{0}\right)^{-1} H^{1 / 2}, \\
H^{3 / 2} & \succ H^{1}\left(H^{1 / 2}\right)^{-1} H^{1} \\
\Delta H^{0} & \succ 0, \quad \Delta H^{1 / 2} \succ 0, \\
\Delta H^{1} & \succ \Delta H^{1 / 2}\left(\Delta H^{0}\right)^{-1} \Delta H^{1 / 2}, \\
\Delta H^{3 / 2} & \succ \Delta H^{1}\left(\Delta H^{1 / 2}\right)^{-1} \Delta H^{1},
\end{aligned}
$$

where we defined $\Delta H_{N}^{\ell} \equiv H_{N-1 / 2}^{\ell}-\hat{s} H_{N}^{\ell+1 / 2}$ and used Schur's complement. The lower label of Hankel matrices is implicit and corresponds to $N$.

All half-integer arcs in these conditions are replaced using Eq. (51),

$$
a_{n+1 / 2}=\frac{2}{2 n+3}\left(a_{n}^{(1)}-\partial_{t} a_{n}\right) .
$$

Together with the positivity bounds for $a_{n}^{(1)}$, involving $a_{n}^{(1)}$ up to $\lfloor N-1 / 2\rfloor$, they define the space of allowed arc derivatives, once we eliminate all the $a_{n}^{(1)}$. 
[1] T. N. Pham and T. N. Truong, Evaluation of the derivative quartic terms of the meson chiral lagrangian from forward dispersion relation, Phys. Rev. D 31, 3027 (1985).

[2] B. Ananthanarayan, D. Toublan, and G. Wanders, Consistency of the chiral pion pion scattering amplitudes with axiomatic constraints, Phys. Rev. D 51, 1093 (1995).

[3] M. R. Pennington and J. Portoles, The chiral Lagrangian parameters, L1, L2, are determined by the rho resonance, Phys. Lett. B 344, 399 (1995).

[4] A. Adams, N. Arkani-Hamed, S. Dubovsky, A. Nicolis, and R. Rattazzi, Causality, analyticity and an IR obstruction to UV completion, J. High Energy Phys. 10 (2006) 014.

[5] B. Bellazzini, Softness and amplitudes? positivity for spinning particles, J. High Energy Phys. 02 (2017) 034.

[6] L. Vecchi, Causal versus analytic constraints on anomalous quartic gauge couplings, J. High Energy Phys. 11 (2007) 054.

[7] A. Nicolis, R. Rattazzi, and E. Trincherini, Energy's and amplitudes' positivity, J. High Energy Phys. 05 (2010) 095; Erratum, J. High Energy Phys. 11 (2011) 128.

[8] C. de Rham, S. Melville, A. J. Tolley, and S.-Y. Zhou, Positivity bounds for scalar field theories, Phys. Rev. D 96, 081702 (2017).

[9] C. de Rham, S. Melville, A. J. Tolley, and S.-Y. Zhou, UV complete me: Positivity bounds for particles with spin, J. High Energy Phys. 03 (2018) 011.

[10] N. Arkani-Hamed and Y.-t. Huang, Respectively, lectures at the CERN winter school on supergravity, strings and gauge theory 2019, in Proceedings at the 24th Rencontres Itzykson of the IPhT of CEA-Saclay (2019).

[11] Z. Komargodski and A. Schwimmer, On renormalization group flows in four dimensions, J. High Energy Phys. 12 (2011) 099.

[12] X. O. Camanho, J. D. Edelstein, J. Maldacena, and A. Zhiboedov, Causality constraints on corrections to the graviton three-point coupling, J. High Energy Phys. 02 (2016) 020.

[13] B. Bellazzini, C. Cheung, and G. N. Remmen, Quantum gravity constraints from unitarity and analyticity, Phys. Rev. D 93, 064076 (2016).

[14] B. Bellazzini, L. Martucci, and R. Torre, Symmetries, sum rules and constraints on effective field theories, J. High Energy Phys. 09 (2014) 100.

[15] B. Bellazzini, F. Riva, J. Serra, and F. Sgarlata, Beyond Positivity Bounds and the Fate of Massive Gravity, Phys. Rev. Lett. 120, 161101 (2018).

[16] C. Cheung and G. N. Remmen, Positive signs in massive gravity, J. High Energy Phys. 04 (2016) 002.

[17] M. A. Luty, J. Polchinski, and R. Rattazzi, The $a$-theorem and the asymptotics of 4D quantum field theory, J. High Energy Phys. 01 (2013) 152.

[18] J. Distler, B. Grinstein, R. A. Porto, and I. Z. Rothstein, Falsifying Models of New Physics via Ww Scattering, Phys. Rev. Lett. 98, 041601 (2007).

[19] C. Englert, G. F. Giudice, A. Greljo, and M. Mccullough, The $\hat{H}$-parameter: An oblique higgs view, J. High Energy Phys. 09 (2019) 041.

[20] B. Bellazzini, F. Riva, J. Serra, and F. Sgarlata, Massive higher spins: Effective theory and consistency, J. High Energy Phys. 10 (2019) 189.
[21] B. Bellazzini, M. Lewandowski, and J. Serra, Positivity of Amplitudes, Weak Gravity Conjecture, and Modified Gravity, Phys. Rev. Lett. 123, 251103 (2019).

[22] W.-M. Chen, Y.-T. Huang, T. Noumi, and C. Wen, Unitarity bounds on charged/neutral state mass ratios, Phys. Rev. D 100, 025016 (2019).

[23] G. N. Remmen and N. L. Rodd, Consistency of the standard model effective field theory, J. High Energy Phys. 12 (2019) 032.

[24] C. Zhang and S.-Y. Zhou, Convex Geometry Perspective to the (Standard Model) Effective Field Theory Space, Phys. Rev. Lett. 125, 201601 (2020).

[25] H. Lehmann, Analytic properties of scattering amplitudes as functions of momentum transfer, Nuovo Cimento 10, 579 (1958).

[26] A. Martin, Extension of the axiomatic analyticity domain of scattering amplitudes by unitarity. 1, Nuovo Cimento A $\mathbf{4 2}$, 930 (1966).

[27] D. Liu, A. Pomarol, R. Rattazzi, and F. Riva, Patterns of strong coupling for Lhc searches, J. High Energy Phys. 11 (2016) 141.

[28] B. Bellazzini, F. Riva, J. Serra, and F. Sgarlata, The other effective fermion compositeness, J. High Energy Phys. 11 (2017) 020.

[29] B. Bellazzini and F. Riva, New phenomenological and theoretical perspective on anomalous $\mathrm{ZZ}$ and $\mathrm{Z}$ ? processes, Phys. Rev. D 98, 095021 (2018).

[30] A. Nicolis, R. Rattazzi, and E. Trincherini, The Galileon as a local modification of gravity, Phys. Rev. D 79, 064036 (2009).

[31] K. Hinterbichler and A. Joyce, Goldstones with extended shift symmetries, Int. J. Mod. Phys. D 23, 1443001 (2014).

[32] A. Martin, Scattering Theory: Unitarity, Analyticity and Crossing, Vol. 3 (1969).

[33] M. Froissart, Asymptotic behavior and subtractions in the Mandelstam representation, Phys. Rev. 123, 1053 (1961).

[34] A. Martin, Unitarity and high-energy behavior of scattering amplitudes, Phys. Rev. 129, 1432 (1963).

[35] M. B. Green and C. Wen, Superstring amplitudes, unitarily, and Hankel determinants of multiple zeta values, J. High Energy Phys. 11 (2019) 079.

[36] E g. see theorem 4.17.4 of, Real Analysis. A Comprehensive Course in Analysis, Part 1 (American Mathematical Society, 2015, Simon, B.).

[37] T. Becher, A. Broggio, and A. Ferroglia, Introduction to soft-collinear effective theory, Lect. Notes Phys. 896, 1 (2015).

[38] G. Goon, K. Hinterbichler, A. Joyce, and M. Trodden, Galileons as Wess-Zumino terms, J. High Energy Phys. 06 (2012) 004.

[39] C. Itzykson and J. B. Zuber, Quantum Field Theory, International Series In Pure and Applied Physics (McGraw-Hill, New York, 1980).

[40] Y.S. Jin and A. Martin, Number of subtractions in fixed-transfer dispersion relations, Phys. Rev. 135, B1375 (1964).

[41] B. Bellazzini, J. Elias Miro, R. Rattazzi, M. Riembau, and F. Riva, Bootstrapping EFTs (to be published). 
[42] Y.-t. Huang, J.-Y. Liu, L. Rodina, and Y. Wang, Carving out the space of open-string S-matrix, J. High Energy Phys. 04 (2021) 195.

[43] M. F. Paulos, J. Penedones, J. Toledo, B. C. van Rees, and P. Vieira, The S-matrix bootstrap III: Higher dimensional amplitudes, J. High Energy Phys. 12 (2019) 040.
[44] A. L. Guerrieri, J. Penedones, and P. Vieira, Bootstrapping QCD Using Pion Scattering Amplitudes, Phys. Rev. Lett. 122, 241604 (2019).

[45] M. Correia, A. Sever, and A. Zhiboedov, An analytical toolkit for the S-matrix bootstrap, arXiv:2006 .08221 . 